\title{
Développement, technologies mobiles et commerce équitable
}

Quand les pratiques numériques mises en place pour les producteurs du Sud inspirent le Nord

Development, mobile technologies and fair trade: when digital practices undertaken for southern producers inspire northern countries Desarrollo, tecnologías móviles y comercio justo : cuando las prácticas digitales emprendidas por los productores del sur inspiran a los países del norte

\section{Hanitra Randrianasolo-Rakotobe et Jean-Michel Ledjou}

\section{(2) OpenEdition}

Édition électronique

URL : http://journals.openedition.org/ctd/647

DOI : $10.4000 /$ ctd. 647

ISSN : 2491-1437

Éditeur

Chaire Unesco Pratiques émergentes en technologies et communication pour le développement

Référence électronique

Hanitra Randrianasolo-Rakotobe et Jean-Michel Ledjou, « Développement, technologies mobiles et commerce équitable », Communication, technologies et développement [En ligne], 6 | 2018, mis en ligne le 18 décembre 2018, consulté le 09 juin 2019. URL : http://journals.openedition.org/ctd/647 ; DOI : $10.4000 /$ ctd. 647

Ce document a été généré automatiquement le 9 juin 2019

Communication, technologies et développement 


\section{Développement, technologies mobiles et commerce équitable}

Quand les pratiques numériques mises en place pour les producteurs du Sud inspirent le Nord

Development, mobile technologies and fair trade: when digital practices

undertaken for southern producers inspire northern countries

Desarrollo, tecnologías móviles y comercio justo : cuando las prácticas digitales

emprendidas por los productores del sur inspiran a los países del norte

Hanitra Randrianasolo-Rakotobe et Jean-Michel Ledjou

\section{Introduction}

1 Le dynamisme du marché des technologies numériques, et singulièrement celui de l'Internet mobile se trouve souligné dans de nombreux rapports publiés par les institutions internationales. Dans ce contexte, deux éléments nous ont amenés à axer notre communication sur un nouveau type de comportement d'achat que l'on peut relier à l'explosion des réseaux mobiles. Ceux-ci ont trait aux formes d'engagement et de participation en ligne dans les pays développés qui, dans un premier temps, ont bénéficié à des producteurs des pays en développement, et depuis 2016, concernent des petits producteurs des pays développés, notamment, français. Dès lors, nous nous posons la question de savoir si nous avons affaire à une innovation inversée, basée sur le numérique, s'appuyant en outre sur une création de valeurs solidaire, annoncée et assumée.

2 Notre communication s'attache à comprendre les particularités d'une telle innovation. Afin d'apporter des éléments de réponse, notre démarche tire profit du croisement de matériels et des méthodes utilisés d'une part en sciences de l'information et de la communication et, d'autre part, en économie. Notre propos est organisé en deux parties. La première dessine le cadre général qui a vu naître l'engagement des consommateurs. Nous l'illustrerons avec le commerce équitable, forme la plus connue de l'économie 
solidaire. Si à l'origine, les actions innovantes en faveur de la lutte contre la pauvreté ont pour cibles les producteurs du Sud et sont portées par des consommateurs militants, il semble que désormais, le consommateur engagé pour cette cause n'ait plus tout à fait le même profil. Nous verrons comment la consommation d'un bien en fonction de sa qualité sociale, comme le paiement d'un prix équitable aux producteurs, ne répond plus uniquement à une démarche militante hors ligne qui nécessitait auparavant un engagement supposant d'appartenir à une association, d'avoir une connaissance de la filière ou une connaissance du terrain. Ainsi nous montrerons que l'internet mobile a contribué à rationaliser cet engagement en faisant naître un nouveau type de comportement.

3 La seconde partie rend compte de l'utilisation des piliers et des caractéristiques technologiques de l'innovation sociale, mais cette fois-ci, au profit de producteurs français. Depuis quelque temps, en effet, le commerce équitable Nord/Nord se développe à partir d'innovations empruntées au commerce équitable nord-sud. En utilisant le dispositif numérique, des entrepreneurs sont arrivés à faire ce que la Commission européenne et le gouvernement français n'ont pu faire, à savoir maintenir un prix de vente à un niveau permettant à des producteurs d'une filière en crise de conserver une activité pérenne. Ce comportement, aujourd'hui quantitativement faible, nous semble porteur de potentiel.

\section{De la lutte contre les inégalités à la consommation responsable connectée}

4 La globalisation de l'économie et la croissance mondiale ont soulevé bien des espoirs sur le plan de l'amélioration durable des conditions de vie et du bien-être, pour l'ensemble de l'humanité, mais surtout pour les millions d'individus parmi les plus pauvres. Dans le domaine social, on assiste à une réduction de l'extrême pauvreté de long terme, cependant on constate en parallèle, un creusement des inégalités qui décuple la vulnérabilité face aux risques d'un monde interconnecté. Certaines populations notamment en Afrique subsaharienne ne tirent pas profit de la mondialisation. Pire, elles se trouvent encore plus vulnérables dans notre monde globalisé.

\section{Justice sociale et globalisation}

5 Afin de réduire la pauvreté, les bienfaits de la participation au commerce international sont plus que jamais avancés. Première source multilatérale d'aide au commerce, le groupe de la Banque mondiale alloue annuellement 15 milliards de dollars de financement depuis 2002. Il s'agit de contribuer à la réalisation des «Objectifs du Millénaire pour le Développement » en facilitant l'accès des pays en développement au marché mondial, en soutenant la mise en place d'un système commercial prévisible et réglementé (BM, 2013). La commission sur la croissance et le développement pilotée par le groupe souligne que tous les pays en développement qui ont connu des périodes durables de croissance économique soutenue ont prospéré en s'ouvrant aux marchés mondiaux. Considéré comme un instrument capable d'aider les pays à tirer parti de la mondialisation, le système commercial est au centre des recherches, des opérations et des financements. 
6 En Afrique subsaharienne où le secteur primaire agricole reste prépondérant, le revenu moyen d'un paysan est bien inférieur au seuil de pauvreté. Pour une culture emblématique comme le cacao, le réseau européen Voice (Alet, 2013) montre qu'il faudrait que les revenus augmentent de $341 \%$ au Ghana, ou de $1608 \%$ en Côte d'Ivoire pour atteindre le seuil de deux dollars par jour. La paupérisation nourrit la vulnérabilité de ces paysans, classés parmi les plus pauvres. Pour contribuer à une réflexion sur la justice sociale au XXIe siècle, l'importance prise par les services dans la création de richesse et l'interdépendance des sociétés nous amène à étudier la place particulière des technologies numériques.

\section{La place des technologies numériques}

7 L'accès aux technologies numériques est une préoccupation de la communauté internationale. C'est un enjeu économique et sociétal jugé primordial pour tous les États, et à fortiori pour les pays en développement. Améliorer l'accès à ces technologies dans les régions les plus pauvres au monde figure à l'agenda international. Ainsi, s'agissant du continent africain, le programme mondial Connecter l'Afrique a vu l'implication des Nations unies, des institutions financières, des gouvernements des entreprises et des citoyens. Sur le continent africain, par exemple, les abonnements aux mobiles n'ont cessé de croître dans la dernière décennie. Début 2012, on dénombre 649 millions de cartes SIM en Afrique ( $31 \%$ de taux de pénétration pour l'Afrique subsaharienne 253 millions d'abonnés uniques), ce qui fait du continent, compte tenu des marges de progression potentielle, un marché bien plus important que celui des États-Unis (350 millions) ou de l'Union européenne (770 millions, taux de pénétration $123 \%$ ). Ce phénomène comporte l'expansion des terminaux mobiles (smartphones et tablettes), dans un contexte où leurs prix diminuent progressivement. Les opérateurs tiennent non seulement compte de la faible bancarisation, mais adaptent aussi leurs offres et leurs services aux spécificités du marché (Ledjou, Randrianasolo, 2013).

8 Si les disparités régionales en matière de taux de pénétration diminuent significativement, l'écart reste important dans le déploiement de l'Internet haut débit. Beaucoup reste donc à faire pour exploiter les technologies numériques. Car si leur succès semble se mesurer à l'aune des abonnements dans les pays en développement; dans les pays développés, celles-ci sont considérées comme des accélérateurs de la croissance, ainsi que l'a prouvé l'ensemble des discours autour de la nouvelle économie, produits depuis les années 1990. Comme le rappelle Pierre Beckouche, «le web 2.0 a ouvert la voie à l'horizontalisation massifiée des relations entre les individus » (Beckouche, 2017). Dans ce cadre, face aux multiples défis du monde contemporain, les technologies numériques ont fortement contribué à la prise de conscience de notre communauté de destin. Sous l'effet de l'interdépendance des sociétés, la question de l'amélioration des conditions de vie des plus vulnérables a changé d'échelle, et ce d'autant plus que l'essor du numérique donne aux populations connectées le sentiment de vivre au sein d'un village global. Ce rétrécissement géographique virtuel traduit en outre une tension entre l'économie sociale et solidaire (ESS) d'une part, et l'économie collaborative d'autre part. Alors que l'ESS prend de l'ampleur dans les pays du Nord, celle-ci, consubstantielle au fonctionnement des pays du Sud, s'attache avant tout à dynamiser les activités de production liées à un territoire spécifique. L'économie collaborative, elle, ne connaît pas de frontières. À travers les plateformes numériques, cette dernière marque notre entrée 
dans l'ère de l'exploitation massive et organisée des données. Pris entre le proche et le lointain, le commerce équitable qui a permis de rapprocher les petits producteurs régionaux des grands marchés nationaux et internationaux n'échappe pas à ces transformations.

\section{Le numérique éveille une nouvelle forme de consommation équitable}

Nous l'avons dit, un consensus émerge sur les bénéfices que les pays en développement ont tirés de leur participation au commerce mondial. L'interdépendance de nos sociétés a, quant à elle, suscité la construction d'une nouvelle qualité sociale des biens produits dans les pays marqués par la pauvreté et les inégalités. Cette qualité sociale prend racine dans les relations tissées entre producteur et consommateur depuis l'avènement du commerce international. La connaissance des conditions de vie des producteurs du Sud n'est pas donc pas un phénomène nouveau. Mouzon (2014) rappelle d'ailleurs que l'invention d'un commerce qui se proclame "éthique» remonte au début du XIXe siècle, quand des boutiques militent contre l'esclavage apparaissent aux États-Unis. À partir du début des années 1960, une nouvelle dynamique, illustrée par le mouvement du commerce équitable, voit le jour. Elle constitue la forme la plus connue et la plus pratiquée de ce que l'on nomme «l'économie solidaire ».

10 Avec comme objectif prioritaire l'amélioration des conditions de vie de producteurs marginalisés, le commerce équitable attend du consommateur des pays développés qu'il fasse le choix d'acheter un paquet de chocolat, de café ou de tout autre produit, non seulement pour les caractéristiques propres à ce bien, mais aussi, en acceptant de payer plus cher pour une caractéristique supplémentaire d'ordre social, celle de " pouvoir faire du bien ». Toute la démarche de sensibilisation vise, par conséquent, à transformer la propension à acheter une qualité sociale en un acte d'achat qui mobilisera généralement le paiement d'un surcoût. Pour le producteur au Sud, cet acte d'achat solidaire permettra de pouvoir choisir de rester producteur et de ne pas avoir à quitter sa terre pour améliorer son revenu et sa qualité de vie (Randrianasolo, Dahmani, Dubois, 2014). Pour Dubigeon (2009), la conscience d'un danger planétaire progresse au sein de nos sociétés interdépendantes. Il attire l'attention sur une exclusion croissante - d'une partie de la population mondiale - génératrice d'une profonde déstabilisation sociale. Le niveau d'accès aux opportunités économiques a certainement augmenté, mais paradoxalement le degré d'inégalité sociale est source de menaces. Rejoignant plusieurs études sur le sujet, l'auteur considère que l'éthique de responsabilité (Randrianasolo, 2006; Ballet et al., 2008) devient la clé du nouveau système sociotechnique qui sous- tend le monde moderne. L'analyse de l'évolution du commerce équitable nous permet de préciser le contour et les impacts de cette responsabilité en même temps que le rôle exercé par les technologies mobiles.

\section{Des technologies mobiles au service des petits producteurs du Sud}

11 Par la recherche de solutions novatrices, l'économie solidaire, s'efforce de combattre les grands problèmes sociaux et environnementaux actuels, qui menacent la durabilité de nos sociétés. "L'information, la diffusion de la connaissance devenant totalement ubiquitaire, non seulement les utilisateurs peuvent bénéficier de conseils appropriés, 
mais peuvent aussi devenir des contributeurs très significatifs de la création d'un bienêtre collectif» (Babinet, 2014, P79). À cet égard, les concepteurs d'applications pour terminaux numériques ont montré qu'il était possible d'informer chaque consommateur, chaque citoyen sur les conséquences de son acte de consommation. Les applications Mesgouts.fr et Noteo, sur lesquelles nous basons notre illustration, offrent de nouvelles opportunités au consommateur souhaitant contribuer à l'amélioration des conditions de vie des producteurs les plus pauvres. Celles-ci donnent, en effet, accès à de nouvelles informations et possibilités d'actions. Il est dorénavant possible de détenir une information traitée sur les impacts sociétaux de produits disponibles en moyennes et grandes surfaces. Il suffit pour ce faire de scanner un code-barres à l'aide d'un smartphone. Sur Noteo, par exemple, des notes sont attribuées aux produits en fonction d'items qui ont trait à la santé, à l'environnement biologique et social. Il s'agit là d'une véritable innovation sur le plan du comportement d'achat.

Disposer de données instantanées et quantifiées sur l'impact de son acte, et ce, quel que soit le lieu d'achat et sans même avoir testé le produit se révèle le moyen d'une prise de conscience collective des conditions de production. En théorie, s'inscrire dans une telle démarche, renforce non seulement la liberté de choix des consommateurs, mais également celle des producteurs. Sur Mesgouts.fr (voir figure), le consommateur indique ce qui est important à ses yeux. L'application lui permet de matérialiser son désir d'agir pour améliorer les conditions sociales des producteurs ou pour protéger l'environnement. L'application retient ces informations, et lorsque le consommateur scanne un produit ou consulte une liste de produits, une note est immédiatement disponible. Une note élevée indique une conformité avec les critères et les valeurs du consommateur. Le nombre d'inscrits participant à cette démarche est mis en valeur par les concepteurs, renforçant par là même le sentiment d'appartenance à un réseau. L'application a par ailleurs construit une note, nommée « Appréciation » qui renseigne la note moyenne donnée à un produit par la communauté. Ainsi, la consommation de biens en fonction de leur qualité sociale ne correspond plus seulement à une démarche militante qui suppose une connaissance de la filière, voire d'être membre d'une association. À un moment où les grandes enseignes de distribution cherchent à tirer profit de cette tendance des sociétés du nord à la consommation équitable, le terminal mobile resserre les liens entre les consommateurs et les différents acteurs du commerce équitable. 
Figure 1. Extrait Application Noteo, exemple de notation d'un chocolat équitable

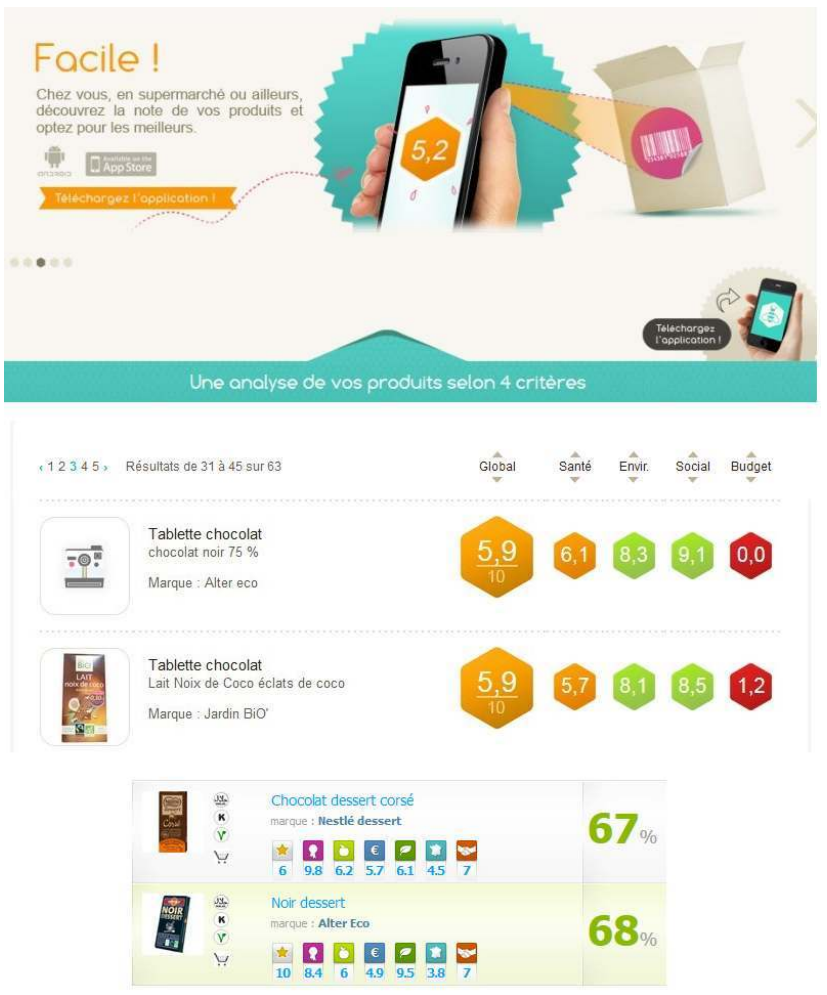

Figure 2. Extrait Application Mesgouts.fr. Exemple de notation, chocolat équitable

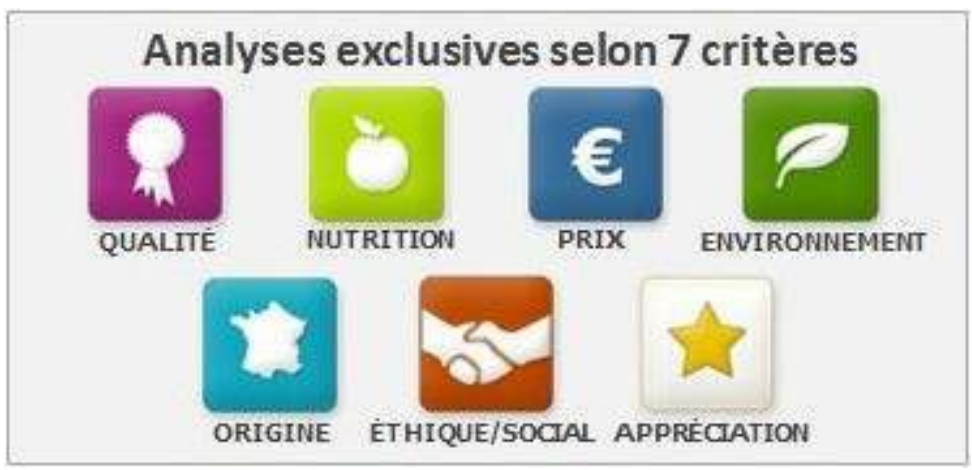

1. Je m'inscris, c'est gratuit

2. J'indique mes valeurs (7 critères disponibles)

3. Je consulte les produits ou je scanne le code-barres

4. Je compare et je choisis en fonction de la note

Dès lors, animés par un désir de formalisation et de mise en relation des faits observés au Sud comme au Nord, avec des connaissances théoriques disponibles, nous pensons que la forme de l'engagement du consommateur à travers les réseaux numériques mobiles met en avant une responsabilité ex ante. 


\section{La responsabilité ex ante une opportunité pour les petits producteurs du Sud}

14 À partir des travaux de plusieurs chercheurs dont ceux d'Hans Jonas, nous constatons que la forme de responsabilité utilisée jusqu'à présent - essentiellement rétroactive - ne rend pas compte des particularités de l'innovation inversée, permise par les technologies numériques, dans le domaine du commerce équitable. L'éthique de la responsabilité classique, c'est-à-dire rétroactive, stipule que la responsabilité est engagée une fois que l'action est faite. C'est une responsabilité que nous devons assumer en fonction de nos actes passés et des conséquences qu'ils ont entraînées. Selon cette figure, si l'être humain n'agit pas, on ne peut lui imputer de responsabilité a posteriori (Métayer, 1997).

Or, dans le cadre de l'explosion des réseaux numériques, l'action peut être interprétée comme le fruit d'une prise de conscience et de responsabilité. Une telle éthique de la responsabilité a été conceptualisée par Hans Jonas depuis 1979. Ce dernier met en exergue une responsabilité ${ }^{1}$ qui n'est pas consécutive à un acte passé, mais qui préexiste à cet acte et peut même être à la source de celui-ci. Elle commande l'acte à accomplir en posant l'acteur correspondant comme responsable de cet acte a priori. Ce qui fonde cette responsabilité, c'est qu'un être vivant se trouve dans notre sphère d'influence et que son sort dépend directement de nous, soit parce qu'il a besoin de nous, soit parce qu'il est menacé par nos actions. C'est la vulnérabilité et la fragilité de cet être qui engage notre responsabilité. Ramenée au commerce équitable, cette approche de la responsabilité conduit à arguer que la vulnérabilité et la fragilité des producteurs marginalisés en Afrique concernent la responsabilité du consommateur: une fois informé des conséquences de son achat via une application de notations, celui-ci engage une dépense.

Source d'action, la responsabilité ex ante est à la base d'un nouveau comportement, désigné par Michelleti sous le vocable "buycott». Ce néologisme fabriqué à partir de l'anglais to buy (acheter), consiste à acheter des produits répondant à des critères sociaux et environnementaux dont il faut organiser la production (De Ravignan, 2004). En tout cas, théoriquement, la mobilisation des travaux sur l'éthique de la responsabilité qui relient liberté et action apporte des éléments qui peuvent rendre compte du mécanisme à la base de ce nouveau type de comportement. Quant au dispositif numérique, il occupe une place déterminante, car c'est à travers lui que s'exercent de nouvelles pratiques, de nouvelles formes de participation qui, en l'espèce, ne se limitent plus au seul commerce équitable Nord/Sud.

\section{Quand des petits producteurs français tirent parti de la responsabilité ex ante}

En 2016, à travers la "marque du consommateur ", émerge en France une innovation sociale consacrant l'intentionnalité et la responsabilité de l'acte de consommation. Cantonnée dans un premier temps au secteur du lait, cette marque veut que les producteurs laitiers soient convenablement rémunérés et qu'à tout le moins ils ne perdent pas d'argent. Sur Internet, la responsabilité ex ante est matérialisée par 4 préférences reliées à différents niveaux de qualité. Dans la conception de son produit idéal, le consommateur connaît le coût supporté par le producteur et doit s'engager à 
payer le prix correspondant. Au final, quel que soit le critère retenu, figure toujours le fait que :

- le producteur ne perde pas d'argent,

- qu'il puisse se payer convenablement,

- et qu'il dispose du temps libre et du remplacement (cf. Figure 3).

Figure 3. La marque du consommateur - Le lait créé par le consommateur

Tous les produits sont directement issus de nos attentes et de nos critères de choix (cf. le lait équitable)

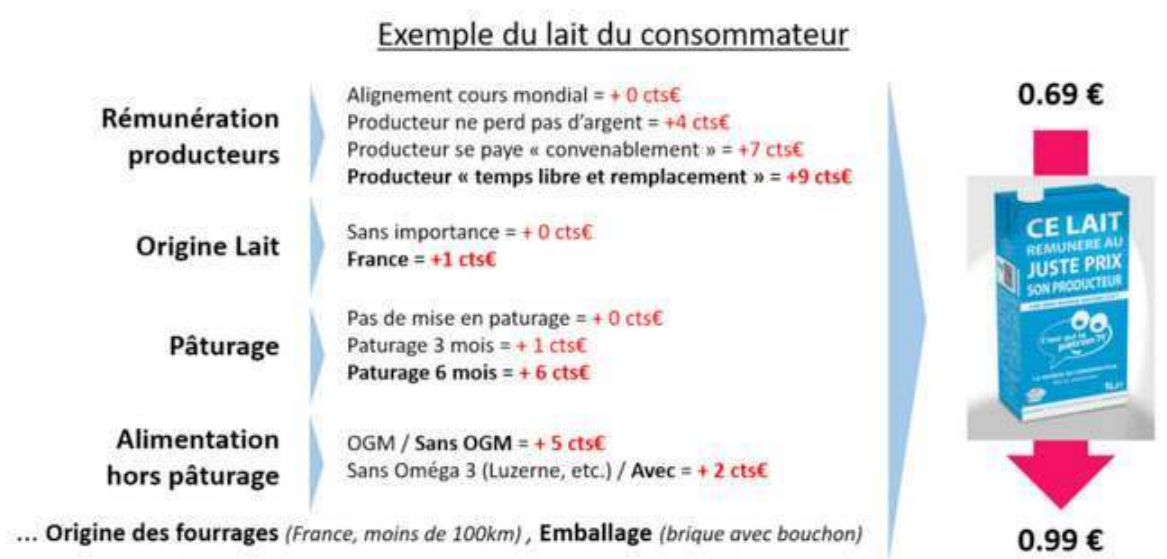

Source : site web officiel, la marque du consommateur, visité le 3 mars 2017

la marque du consommateur. La groupe de distribution Carrefour ${ }^{2}$, qui figure parmi les parties prenantes de cette initiative, précise que c'est la première marque où le prix et les critères de qualité sont fixés par les consommateurs. De son côté, la marque du consommateur promeut le fait que le lait équitable rémunère son producteur au juste prix. L'engagement repose sur 7850

19 "consom'acteurs» qui soutiennent 51 éleveurs dans l'Ain. La tendance est quantitativement faible! La veille et l'histoire économique du lait équitable vont cependant renforcer le potentiel qui s'est dégagé de la conceptualisation de la « consom'action ». En tout état de cause, l'innovation sociale inversée décrite ici porte en elle-même les ferments d'une économie plus solidaire fondée sur les besoins de producteurs vulnérables.

\section{Conclusion}

En agrégeant des consommateurs mus par les mêmes intérêts, le numérique a incontestablement fourni une plus grande visibilité aux producteurs vulnérables issus des pays du Sud et du Nord. Il a par ailleurs permis d'appliquer en France une innovation sociale jusque-là limitée au seul commerce équitable, à savoir la fixation d'un prix donnant aux producteurs vulnérables la possibilité de vivre du fruit de leur travail. En outre, du fait de leur fonctionnement bottom- up, les technologies numériques mettent une multitude d'informations à la disposition des consommateurs. Ce faisant, celles-ci renforcent la responsabilité ex ante. Il convient cependant d'être prudent. Car loin d'être 
la panacée, les technologies peuvent constituer une menace pour les petits producteurs: à l'heure de la notation généralisée, des « like » et autres formes d'appréciation, c'est la réputation de toute une filière qui est en jeu. Enfin, notons que la notion de confiance est des plus importantes pour le changement d'échelles de ces nouveaux comportements digitaux au profit de l'amélioration des conditions de vie des plus pauvres. La question de la méthodologie de la notation, de la participation des populations représentées par le critère « social ", tout comme la récupération de la démarche pour asseoir le pouvoir de marché des grands acteurs sont également des aspects qu'il s'agit de prendre en compte.

\section{BIBLIOGRAPHIE}

African Development Bank (Afdb), Évaluation des avancées en direction des objectifs du Sommet Connecter l'Afrique, Rapport complet, Ed : Groupe Banque mondiale, Washington, 2013.

Alain Kiyindou, Quand l'Afrique réinvente la téléphonie mobile, L'Harmattan, Paris, 2015.

Amina Bécheur, Nil Toulouse, Le commerce équitable. Entre utopie et marché, Vuibert, Paris, 2008.

Annie Chéneau Loquay, Modes d'appropriation innovants de la téléphonie mobile en Afrique, MAEE, UIT, Paris, 2010.

Antoine De Ravignan, «Carrefour, la tactique de l'éthique », Alternatives Internationales, $\mathrm{n}^{\circ} 18$, 2004, pp. 34-35.

Cécile Méadel, « Les savoirs profanes et l'intelligence du Web », Hermès, n 57, 2010, pp. 111-117.

Céline Mouzon, Consommer autrement, Alternatives économiques Scop-SA, Paris, 2014.

Dominique Pasquier, «Les jugements profanes en ligne sous le regard des sciences sociales », Réseaux, $\mathrm{n}^{\circ} 183,2014$, pp. 9-25.

Gilles Babin, L'ère numérique, un nouvel âge de l'humanité, Le passeur, Paris, 2014.

GSMA, The mobile economy, sub-saharan Africa, 2014. [en ligne] https://www.gsma.com/ mobileeconomy/archive/GSMA_ME_SubSaharanAfrica_2014.pdf (consulté le 22 juillet 2016).

Hanitra Randrianasolo-Rakotobe, Ahmed Dahmani, Jean-Luc Dubois, « De la nécessité d'étendre les références éthiques de l'approche par les capabilités. Enseignements tirés des pratiques du commerce équitable (Accent sur les TIC) », Mondes en développement, $\mathrm{n}^{\circ}$ 168, 2014, pp. 59-76

Hanitra Randrianasolo-Rakotobe, Delphine Caroux, Michel Dubois, Agriculture innovante et dépendance au sentier, WP, Institut polytechnique LaSalle Beauvais, 2015.

Jean-Michel Ledjou, Hanitra Randrianasolo (dir.), Des réseaux et des hommes. Les Suds à l'heure des TIC, Karthala, Paris, 2013.

Michel Métayer, La philosophie éthique, enjeux et débats actuels, Édition ERPI, Québec, 1997.

Michele Micheletti, «Shopping with and for Virtues », In Michele Micheletti (dir.) Political virtue and shopping- individuals, consumerism, and collective action, Palgrave McMillan, New York, 2003, pp. 149-168 
Organisation internationale de la Francophonie, Isoc, Rapportsur la Francophonie numérique, 2014.

Pierre Beckouche, «La révolution numérique est-elle un tournant anthropologique ? ", Le Débat, n 193, 2017, pp. 153-156.

\section{NOTES}

1. Sur ce point on pourra lire avec profit : Randrianasolo, Dubois et Dahmani (2014)

2. https://www.carrefour.fr/, visité le 12 avril 2017

\section{RÉSUMÉS}

En rendant accessibles des informations sur les conséquences sociales d'un achat, les technologies mobiles contribuent à l'exercice de la responsabilité ex ante du consommateur. Dans le domaine du commerce équitable, cette dernière correspond au paiement d'une qualité sociale. Aussi, si cette forme de commerce s'est d'abord déployée au profit exclusif des producteurs des pays en développement, celle- ci concerne, depuis 2014, des producteurs français.

Dans ce contexte, les technologies numériques ont permis aux consommateurs français de révéler leurs préférences et de fixer un prix qui a contribué à lancer la première marque de lait équitable.

In making the informations on the consequences of a purchase available, mobile technologies contribute in the consumer's exercice of ex ante responsibility. As far as Fair trade is concerned, the latter corresponds to the payment of a social quality. Also, although Fair Trade was first deployed for the exclusive benefit of producers in developing countries, since 2014 it has been dealing with French producers. In this context, digital technologies allowed French consumers to reveal their preferences and set a price that helped launch the first fair milk brand.

Permitiendo el acceso a las informaciones sobre las consecuencias sociales de la compra, las tecnologías móviles, contribuyen al ejecicio de la responsabilidad de antemano del consumidor. En el campo del comercio justo, éste corresponde al pago de una calidad social. Si esta forma de comercio al pricipio de su creacion beneficio unicamente a los productores de los paises en vias de desarrollo, desde el 2014 este comercio impacta a los productores franceses. En este contexto, las tecnologías numéricas han permitido a los consumidores franceses de elevar sus preferencias y de fijar un precio que ha contribuido a lanzar la primera marca de leche de comercio justo.

\section{INDEX}

Mots-clés : développement social, réseaux numériques, engagement, innovation sociale inversée, développement durable

Palabras claves : desarrollo social, redes digitales, compromiso, desarrollo sostenible Keywords : social development, digital network, agency, reversed social innovation, sustainable development 


\section{AUTEURS}

\section{HANITRA RANDRIANASOLO-RAKOTOBE}

Centres d'études interdisciplinaires, IDEST-CEI, Université Paris Sud, France JEAN-MICHEL LEDJOU

Centres d'études interdisciplinaires, IDEST-CEI, Université Paris Sud, France 